\title{
Nonlinear Perturbations of the Kaluza-Klein Monopole
}

\author{
Piotr Bizoń, ${ }^{1,2}$ Tadeusz Chmaj, ${ }^{3}$ and Gary Gibbons ${ }^{4}$ \\ ${ }^{1}$ M. Smoluchowski Institute of Physics, Jagiellonian University, Kraków, Poland \\ ${ }^{2}$ Max-Planck-Institut für Gravitationsphysik, Albert-Einstein-Institut, Golm, Germany \\ ${ }^{3}$ H. Niewodniczanski Institute of Nuclear Physics, Polish Academy of Sciences, Kraków, Poland \\ ${ }^{4}$ Department of Applied Mathematics and Theoretical Physics, Cambridge University, Cambridge, United Kingdom
}

(Received 10 April 2006; published 16 June 2006)

\begin{abstract}
We consider the nonlinear stability of the Kaluza-Klein monopole viewed as the static solution of the five-dimensional vacuum Einstein equations. Using both numerical and analytical methods, we give evidence that the Kaluza-Klein monopole is asymptotically stable within the cohomogeneity-two biaxial Bianchi type-IX ansatz recently introduced by Bizoń, Chmaj, and Schmidt [Phys. Rev. Lett. 95, 071102 (2005)]. We also show that for sufficiently large perturbations the Kaluza-Klein monopole loses stability and collapses to a Kaluza-Klein black hole. The relevance of our results for the stability of Bogomol'nyiPrasad-Sommerfield states in M or string theory is briefly discussed.
\end{abstract}

DOI: $10.1103 /$ PhysRevLett.96.231103

PACS numbers: $04.50 .+\mathrm{h}$

Introduction. - The Taub-NUT instanton is the complete Riemannian Ricci-flat 4-manifold with the metric [1]

$$
\begin{aligned}
d s^{2}= & \left(1+\frac{m}{\rho}\right) d \rho^{2}+\left(1+\frac{m}{\rho}\right) \rho^{2}\left(d \theta^{2}+\sin ^{2} \theta d \phi^{2}\right) \\
& +m^{2}\left(1+\frac{m}{\rho}\right)^{-1}(d \psi+\cos \theta d \phi)^{2},
\end{aligned}
$$

where $\rho \geq 0,0 \leq \theta \leq \pi, 0 \leq \phi \leq 2 \pi, 0 \leq \psi \leq 4 \pi$, and $m>0$ is a free parameter which sets the scale. The TaubNUT instanton is topologically $R^{4}$ with the surfaces of constant $\rho$ being the squashed three-spheres. Although complete, the metric (1) is not asymptotically Euclidean, because the $S^{1}$ fibers approach a constant circumference at infinity (rather than growing like $\rho$ ). This kind of asymptotic behavior is called asymptotically locally flat.

By adding the trivial term $-d t^{2}$, one obtains from (1) the regular static solution of the five-dimensional vacuum Einstein equations

$$
\begin{aligned}
d s^{2}= & -d t^{2}+\left(1+\frac{m}{\rho}\right) d \rho^{2}+\left(1+\frac{m}{\rho}\right) \rho^{2}\left(d \theta^{2}+\sin ^{2} \theta d \phi^{2}\right) \\
& +m^{2}\left(1+\frac{m}{\rho}\right)^{-1}(d \psi+\cos \theta d \phi)^{2} .
\end{aligned}
$$

This metric has been used in the past to obtain the fourdimensional magnetic monopole via a Kaluza-Klein reduction along the coordinate $\psi$ [2], and since then it is usually called the Kaluza-Klein (KK) monopole. To the best of our knowledge, the role of the KK monopole in the dynamics of nonasymptotically flat initial data, and, in particular, its nonlinear stability, has not been studied, probably because this problem appeared to require an analysis of the full five-dimensional Einstein equations which is beyond currently available numerical tools, let alone the analytic ones. This situation has changed recently with the introduction of a new cohomogeneity-two symmetry reduction of the five-dimensional vacuum Einstein equations (referred to below as the BCS ansatz [3]) which provided a framework for investigating the dynamics in a simple $1+$ 1-dimensional setting. Since the KK monopole falls within the BCS ansatz, the question of its nonlinear stability now becomes tractable and is the subject of this Letter.

The KK monopole plays an important role in $\mathrm{M}$ or string theory. For example, its metric product with six flat Euclidean dimensions gives a Ricci-flat 11-dimensional Lorentzian metric, which when reduced to ten spacetime dimensions may be interpreted as a D6-brane solution of type IIA string theory [4]. As such, it is an example of what is called a supersymmetric or Bogomol'nyi-PrasadSommerfield (BPS) solution. BPS solutions of supergravity theories are believed by many string theorists to be absolutely stable, both classically and quantum mechanically. However, until now, this belief has never been tested in a fully nonlinear setting. Our results show that the classical stability is not absolute since for sufficiently large perturbations a gravitational collapse to a black hole is possible. The implications of our results for BPS states will be discussed briefly in the conclusions.

Preliminaries. - The BCS ansatz has the form [3]

$$
\begin{aligned}
d s^{2}= & -A e^{-2 \delta} d t^{2}+A^{-1} d r^{2}+\frac{1}{4} r^{2} e^{2 B}\left(d \theta^{2}+\sin ^{2} \theta d \phi^{2}\right) \\
& +\frac{1}{4} r^{2} e^{-4 B}(d \psi+\cos \theta d \phi)^{2},
\end{aligned}
$$

where $A, \delta$, and $B$ are functions of $t$ and $r$. In contrast to spherical symmetry, this ansatz possesses a dynamical degree of freedom, the field $B$, which measures the deformation of sphericity of the angular part of the metric. Substituting the metric (3) into the vacuum Einstein equations, one gets the following system of equations:

$$
A^{\prime}=-\frac{2 A}{r}+\frac{2}{3 r}\left(4 e^{-2 B}-e^{-8 B}\right)-2 r\left(e^{2 \delta} A^{-1} \dot{B}^{2}+A B^{2}\right),
$$




$$
\begin{gathered}
\delta^{\prime}=-2 r\left(e^{2 \delta} A^{-2} \dot{B}^{2}+B^{\prime 2}\right), \\
\left(e^{\delta} A^{-1} r^{3} \dot{B}\right)^{\cdot}-\left(e^{-\delta} A r^{3} B^{\prime}\right)^{\prime}+\frac{4}{3} e^{-\delta} r\left(e^{-2 B}-e^{-8 B}\right)=0,
\end{gathered}
$$

where primes and dots denote derivatives with respect to $r$ and $t$, respectively. The initial value problem for this system was investigated in Ref. [3] in the case of asymptotically flat initial data.

The KK monopole (2) expressed by the ansatz (3) takes the form

$$
B_{0}=\frac{1}{3} \ln \left(1+\frac{\rho}{m}\right), \quad A_{0}=\frac{\left(1+\frac{4 \rho}{3 m}\right)^{2}}{\left(1+\frac{\rho}{m}\right)^{8 / 3}}, \quad e^{2 \delta_{0}}=A_{0},
$$

where

$$
r=2 m^{1 / 2} \rho^{1 / 2}\left(1+\frac{\rho}{m}\right)^{1 / 6} .
$$

Note that scaling invariance excludes the existence of nontrivial solutions which are everywhere regular and asymptotically flat. The KK monopole is the unique nontrivial regular static solution of the system (4)-(6). To see this, note that static solutions which are analytic at the origin form a one-parameter family with the following asymptotic behavior for $r \rightarrow 0$ :

$$
B(r) \sim b r^{2}, \quad A(r) \sim 1-4 b^{2} r^{4}, \quad \delta \sim-2 b^{2} r^{4},
$$

where $b$ is the shooting parameter. Since the system (4)(6) is scale invariant, it follows from the uniqueness of solutions of ordinary differential equations that, up to scaling, there is only one static solution which is analytic at the origin. It is easy to verify that the solution (7) satisfies the regularity conditions (9) with $b=1 /\left(12 m^{2}\right)$. For convenience, hereafter we choose units in which $m=1$.

Linear stability. - Before discussing numerical results, we first demonstrate that the KK monopole is linearly stable within our ansatz. To this end, we seek solutions of the system (4)-(6) in the form

$$
\begin{aligned}
& B(t, r)=B_{0}(\rho)+B_{1}(t, \rho), \\
& A(t, r)=A_{0}(\rho)+A_{1}(t, \rho), \\
& \delta(t, r)=\delta_{0}(\rho)+\delta_{1}(t, \rho),
\end{aligned}
$$

where $\rho$ is a function of $r$ determined implicitly by (8). Substituting (10) into (4)-(6), linearizing and separating the time dependence $B_{1}(t, \rho)=\exp (-i \lambda t) v_{\lambda}(\rho)$, we get the eigenvalue equation for the spectrum of small perturbations around the KK monopole

$$
\begin{aligned}
\mathcal{A} v_{\lambda} & =\lambda^{2} v_{\lambda}, \\
\mathcal{A} & =-\frac{1}{\rho(1+\rho)} \frac{d}{d \rho}\left(\rho^{2} \frac{d}{d \rho}\right)+\frac{18}{\rho(1+\rho)(3+4 \rho)^{2}} .
\end{aligned}
$$

Although this equation cannot be solved analytically in general, there is an explicit solution for $\lambda=0$

$$
v_{0}=\frac{\rho}{3+4 \rho} .
$$

This is the zero mode corresponding to the scaling freedom-it can be obtained by the action of the scaling generator $r \frac{d}{d r}$ on $B_{0}(\rho(r))$. The zero mode (12) has no zeros, which implies by the standard Sturm-Liouville theory that the operator $\mathcal{A}$ has no negative eigenvalues. Thus, the KK monopole is linearly stable within our ansatz. Note that the zero mode is not a genuine eigenfunction because it is not square integrable. More precisely, on the Hilbert space $L^{2}([0, \infty), \rho(1+\rho) d \rho)$, the self-adjoint operator $\mathcal{A}$ has the purely continuous spectrum $\lambda^{2} \in[0, \infty)$.

Nota bene, the Taub-NUT instanton (1) is known to be linearly stable against transverse traceless perturbations. This follows from the work of Hawking and Pope [5], who showed, using two linearly independent covariantly constant spinors which the Taub-NUT metric admits, that the spectrum of the Lichnerowicz operator, which governs the linearized transverse traceless perturbations, is the same (apart from zero modes) as for the scalar Laplacian and therefore non-negative.

Numerical results. - Using a fourth-order accurate finite difference code, we have solved the system (4)-(6) numerically for several families of regular initial data which represent various perturbations of the KK monopole. The overall picture does not depend on the specific choice of a family. The results shown below were produced for initial data of the form (using $P=e^{\delta} A^{-1} \dot{B}$ )

$$
B(0, r)=B_{0}(\rho(r)), \quad P(0, r)=p\left(\frac{r}{R}\right)^{4} e^{-(r-R)^{4} / s^{4}},
$$

where the amplitude $p$ was varied and the parameters $R$ and $s$ were kept fixed. Note that, although the perturbation (13) is exponentially localized, the induced perturbation of the function $A$ has a $1 / r^{2}$ tail as follows from the Hamiltonian constraint (4).

Small perturbations. - We have found that for small amplitudes $p$ the perturbation is scattered off to infinity and the solution returns to equilibrium; i.e., it settles down to the KK monopole with the same parameter $m$. This is shown in Fig. 1. One might wonder why the parameter $m$ does not change under perturbation. The reason is simple: The perturbations considered by us have finite energy, while a change of $m$ would require infinite energy. Here by energy we mean the energy measured with respect to a given KK reference background as described by Deser and Soldate [6]. This energy is determined by the next to 


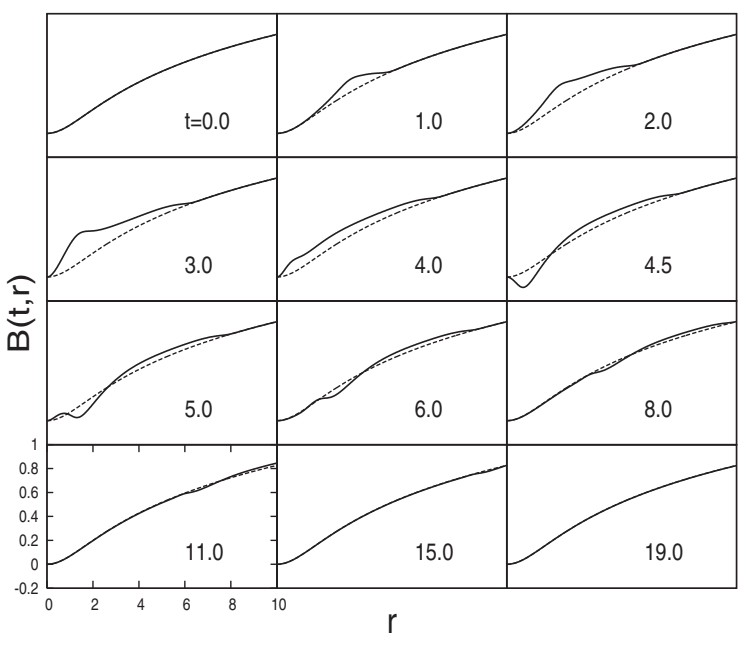

FIG. 1. Asymptotic stability of the Kaluza-Klein monopole. For initial data (13) with a small amplitude $(p=0.1, R=3, s=$ 1 ), we plot a series of snapshots of the function $B(t, r)$ (where $t$ is central proper time). The dashed line shows the unperturbed KK monopole. During the evolution, the excess energy of the perturbation is clearly seen to be radiated away to infinity and the solution returns to equilibrium.

leading order term in the asymptotic expansion at spatial infinity.

The qualitative picture of pointwise convergence to the KK monopole is shown in Fig. 2. The details of this process, in particular, the role of quasinormal modes and the rate of decay of a tail, will be pursued elsewhere.

We have found that a sufficiently strong kick may destabilize the KK monopole. In order to understand what is the fate of a strongly perturbed KK monopole, we need to make a digression.

Kaluza-Klein black holes. -The KK monopole (2) is a special case of the two-parameter family of solutions of the vacuum Einstein equations in five dimensions [7]

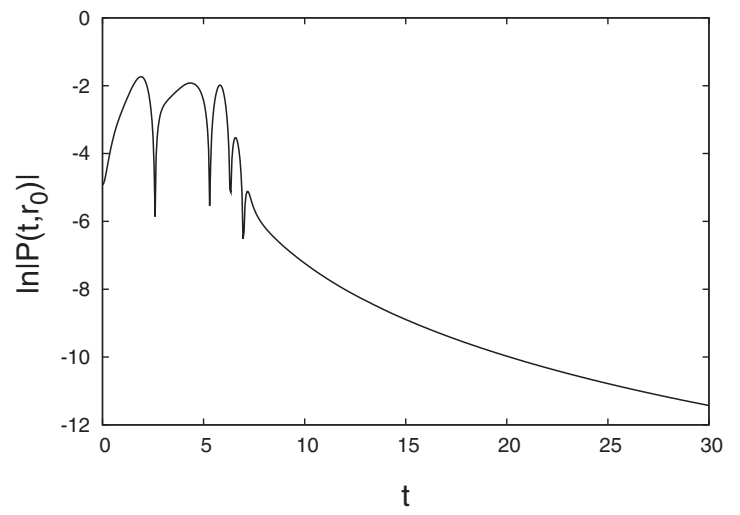

FIG. 2. The convergence to the Kaluza-Klein monopole. For the same initial data as in Fig. 1, we plot the time series $\ln \left|P\left(t, r_{0}\right)\right|$ at $r_{0}=2$. The asymptotic power-law tail is seen at late times.

$$
\begin{aligned}
d s^{2}= & -\frac{\rho}{\Delta} d t^{2}+\frac{\Sigma}{\rho} d \rho^{2}+\Delta \Sigma\left(d \theta^{2}+\sin ^{2} \theta d \phi^{2}\right) \\
& +4 P^{2} \frac{\Delta}{\Sigma}(d \psi+\cos \theta d \phi)^{2},
\end{aligned}
$$

where

$$
\begin{aligned}
& \Delta=\rho+3 M-\sqrt{M^{2}+2 P^{2}}, \\
& \Sigma=\rho+M+\sqrt{M^{2}+2 P^{2}} .
\end{aligned}
$$

Here $\rho \geq 0$, the parameter $M$ is positive, and the parameter $P$ (usually called the magnetic charge) has the range $0<$ $P \leq 2 M$. For $P<2 M$, the metric (14) has a regular horizon at $\rho=0$, so after Ref. [7] we shall refer to this solution as the Kaluza-Klein black hole. For $P=2 M$, the horizon degenerates to a point and one gets the KK monopole (2) with $m=4 M$.

Translating (14) into the ansatz (3), we obtain

$$
B=\frac{1}{3} \ln \left(\frac{\Sigma}{2 P}\right), \quad A=\frac{\rho r^{2}(3 \Sigma+\Delta)^{2}}{36 \Delta^{2} \Sigma^{3}}, \quad e^{2 \delta}=\frac{\Delta}{\rho} A,
$$

where $r=4^{2 / 3} P^{1 / 3} \Sigma^{1 / 6} \Delta^{1 / 2}$. Note that the leading order asymptotic behavior for large $r$ does not depend on $M$ and is determined only by $P$

$$
A \sim \frac{4^{3} P}{9 r}, \quad e^{2 B} \sim \frac{r}{4 P} .
$$

Although the KK black holes are known in closed form, for our purposes it is helpful to look at them from the viewpoint of the shooting procedure. Assuming that there is a nondegenerate horizon at $r=r_{H}$, we obtain from (4)-(6) the following behavior:

$$
\begin{aligned}
& B(r) \sim \beta+\frac{2\left(e^{-2 \beta}-e^{-8 \beta}\right)}{4 e^{-2 \beta}-e^{-8 \beta}}\left(\frac{r}{r_{H}}-1\right), \\
& A(r) \sim \frac{2}{3}\left(4 e^{-2 \beta}-e^{-8 \beta}\right)\left(\frac{r}{r_{H}}-1\right) ;
\end{aligned}
$$

hence, for each $r_{H}>0$ there is a one-parameter family of local solutions with a regular horizon parametrized by $\beta=$ $B\left(r_{H}\right)>0$. Shooting these local solutions towards infinity, we get the KK black holes (15) where

$$
\begin{aligned}
\beta & =\frac{1}{3} \ln \frac{M+\sqrt{M^{2}+2 P^{2}}}{2 P}, \\
r_{H}^{6} & =4^{4} P^{2}\left(M+\sqrt{M^{2}+2 P^{2}}\right)\left(3 M-\sqrt{M^{2}+2 P^{2}}\right)^{3} .
\end{aligned}
$$

Large perturbations. - Now we return to the discussion of numerical results. We have found that for sufficiently large perturbations the inner part of the KK monopole collapses and a horizon forms at some $r=r_{H}>0$. Outside the horizon, the solution settles down to the KK black hole (see Fig. 3). The perturbations considered by us have finite energy, and, therefore, they do not change the leading order asymptotic behavior (16). In this sense, the 


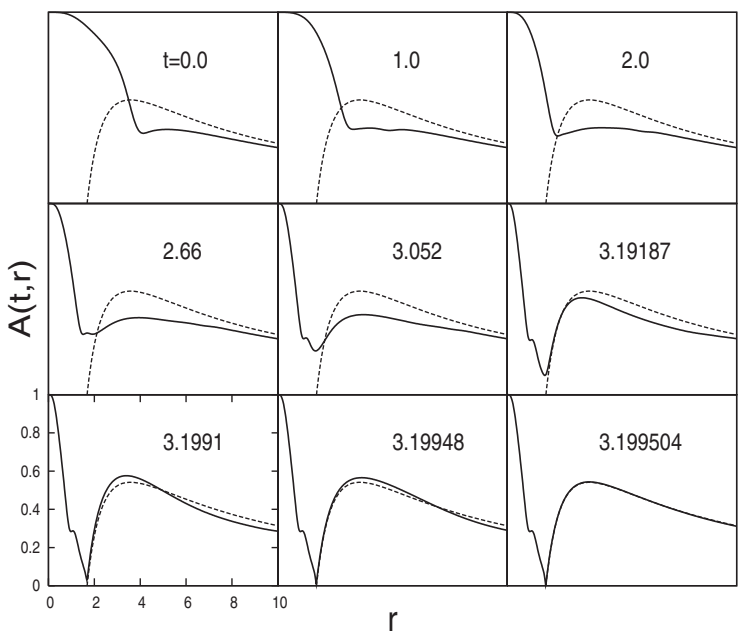

FIG. 3. Instability of the Kaluza-Klein monopole for large perturbations. For initial data (13) with a large amplitude ( $p=$ $0.3, R=3, s=1$ ), we plot a series of snapshots of the function $A(t, r)$ (where $t$ is central proper time). During the evolution, $A(t, r)$ drops to zero at $r=r_{H} \approx 1.68$, which signals the formation of an apparent horizon there. Outside the horizon, the solution relaxes to the Kaluza-Klein black hole (15) (shown by the dashed line) with $M=0.502$.

magnetic charge $P$ can be viewed as the constant of motion. For the KK monopole, $P=2 M=m / 2$, so all our configurations have the same magnetic charge $P=1 / 2$ (recall that we use units in which $m=1$ ) and the end states of evolution differ only by $M$.

As we approach the threshold of collapse from above, the parameter $M$ decreases towards the limiting value $M=$ $1 / 4$ corresponding to the KK monopole. At the same time, as follows from (18), both $\beta$ and $r_{H}$ tend to zero. In this limit, close to the horizon the KK black hole is very well approximated by the Schwarzschild black hole. Near the threshold, we observe the type II discretely self-similar critical behavior governed by the same critical solution as in the asymptotically flat situation [3] (see Fig. 4). This is not surprising in view of the fact that critical collapse is a local phenomenon and, as such, does not depend on the far field behavior.

Conclusions. - We have shown that, although it is classically stable against small perturbations, the KK monopole is classically unstable against perturbations which are large enough to allow gravitational collapse to form a black hole. Classically, the black hole is probably stable. Quantum mechanically, one expects it to emit Hawking radiation, which can carry off energy but not magnetic charge. In the long run, therefore, one might expect the system to settle down to the original KK monopole. If this is so, then the common intuition about BPS states would be correct, provided one bears in mind that the stability can hold only by virtue of quantum mechanical effects.

Finally, we remark that, besides the KK monopole, the BCS ansatz incorporates other nonasymptotically flat so-

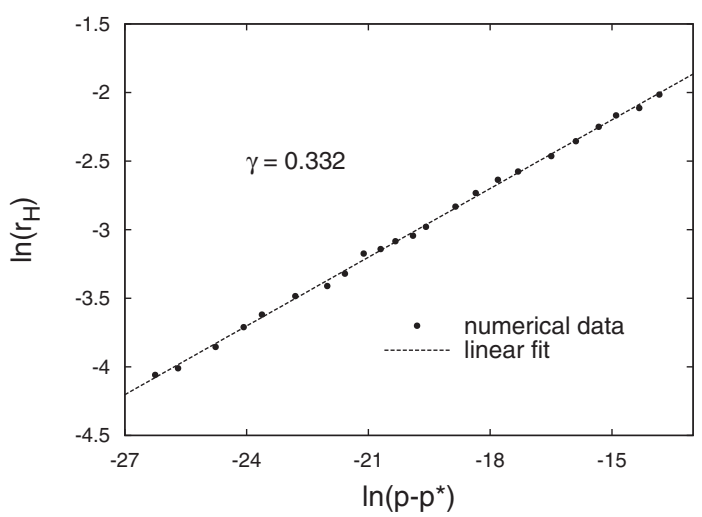

FIG. 4. Critical behavior. For supercritical solutions, we plot the horizon radius vs the distance to the threshold on the log-log scale. The fit of the power law $\ln \left(r_{H}\right)=(\gamma / 2) \ln \left(p-p^{*}\right)+$ const yields $\gamma \approx 0.33$, which agrees (up to numerical errors) with the critical exponent obtained in Ref. [3]. The echoing period $\Delta \approx 0.47$ (read off from the period of wiggles around the linear fit above and computed independently from the spatial shift between the echoes) is also the same.

lutions. In particular, an exact regular time dependent solution is known [8]

$$
\begin{aligned}
d s^{2}= & -d t^{2}+\left(\frac{t}{m}+\frac{m}{\rho}\right) d \rho^{2}+\left(\frac{t}{m}+\frac{m}{\rho}\right) \rho^{2}\left(d \theta^{2}\right. \\
& \left.+\sin ^{2} \theta d \phi^{2}\right)+m^{2}\left(\frac{t}{m}+\frac{m}{\rho}\right)^{-1}(d \psi+\cos \theta d \phi)^{2}
\end{aligned}
$$

This solution can be viewed as the time evolving $\mathrm{KK}$ monopole whose $S^{1}$ fibers pinch off to a point for $t \rightarrow \infty$. It would be interesting to determine the role of this solution in dynamics.

P. B. acknowledges the hospitality of the Isaac Newton Institute in Cambridge during work on this Letter. The research of P. B. and T.C. was supported in part by the Polish Ministry of Science Grant No. 1 PO3B 01229.

[1] S. W. Hawking, Phys. Lett. 60A, 81 (1977).

[2] R. D. Sorkin, Phys. Rev. Lett. 51, 87 (1983); D. J. Gross and M. J. Perry, Nucl. Phys. B226, 29 (1983).

[3] P. Bizoń, T. Chmaj, and B. G. Schmidt, Phys. Rev. Lett. 95, 071102 (2005).

[4] P. K. Townsend, Phys. Lett. B 350, 184 (1995).

[5] S. W. Hawking and C. N. Pope, Nucl. Phys. B146, 381 (1978).

[6] S. Deser and M. Soldate, Nucl. Phys. B311, 739 (1989).

[7] G. W. Gibbons and D. L. Wiltshire, Ann. Phys. (N.Y.) 167, 201 (1985); 176, 393(E) (1987).

[8] G. W. Gibbons, H. Lü, and C. N. Pope, Phys. Rev. Lett. 94, 131602 (2005). 